\title{
Gravitation and energy-momentum conservation in nonsingular general relativity
}

\author{
N. Tsitverblit* \\ School of Mechanical Engineering, Tel-Aviv University, Ramat-Aviv 69978, Israel
}

April 14, 2018

\begin{abstract}
Forced to be flat when nonsingularly described by the equations of general relativity in a synchronous frame, a region outside matter sources is recognized to fail in defining space-time curvature consistently with the material nature of the Lorentz transformation. The curvature then extends to such a region only when there is a continuous background medium there. Arising from vacuum decay in the universe, as any matter, this medium is thus capable of avoiding the singularity of gravitational collapse as well. The theory of general relativity is then suggested to stem from such a formulation of the generalized postulate of relativity as also includes the covariance of energy-momentum conservation for a macroscopically continuous material system, namely the universe. Implying identity between gravitation and inertia, this formulation does not need the principle of equivalence as a separate postulate. The Einstein tensor is thus interpretable as standing for the energy-momentum of the gravitational field. In terms of the background medium, its small-amplitude approximation underlain by matter dynamics and phase transitions also describes what is viewable as gravitational waves. In the framework of such a macroscopic interpretation, gravitation ought to be irrelevant to purely microscopic interactions.
\end{abstract}

\section{Introduction}

Ever since the theory of general relativity was completed $[1,2]$, its commonly recognized aesthetic and conceptual appeal has been vitiated by mathematical issues whose implied potential resolutions have been far short of the high scientific standard otherwise suggested by this theory. Disapproved by Levi-Civita [3] and Lorentz [4] practically immediately after the final version of the Einstein field equations emerged, the pseudotensorial character of energymomentum conservation formulated in $[1,2]$ is one of these issues. A source of concern for other scientists as well $[5,6,7]$, the viability of such a formulation has still been defended by its author with his conceptual interpretations [7, 8, 9, 10, 11].

Einstein's treatment of the issue of energy-momentum conservation has then become largely accepted $[7,11,12,13,14,15]$. It has typically been attributed to what is construed as a principal nonlocalizability of gravitational energy-momentum resulting from local application of the equivalence principle $[12,16]$ in the absence of a natural background space-time structure [15]. Such a perspective has thus also lead either to other suggestions of the same kind $[16,17,18,19$, $20]$ or to other ambiguities when strategies to avoid pseudotensors were adopted [16, 18, 20]. Implying the existence of a curved empty space-time, however, the equivalence principle [21] is just a heuristic hypothesis based on such an aspect of gravity as independence of the gravitationally caused acceleration of the accelerated mass. The concept of energy-momentum conservation then looks much more general and fundamental than such a phenomenologically postulated relation between gravitation and inertia as the equivalence principle [22].

*Address for correspondence: 1 Yanosh Korchak Street, apt. 6, Netanya 42495, Israel; e-mail: tsitver@gmail.com. Most of this study was accomplished at the above Tel-Aviv University address. 
Without later observational discoveries, in particular, introduction of the equivalence principle to explain gravitation has also been motivated by the associated extension of the original principle of relativity to noninertial reference frames $[13,16,18,19,21]$. Thus referred to as the generalized principle of relativity, this extension is what actually underlies the invariance of the Einstein field equations. Initially distinguished by humans, however, such a concept as frame noninertiality could hardly affect the phenomena occurring in nature. From this perspective, the existence of a mathematical description of all these phenomena that is independent of any reference frame would a priori have to be legitimately anticipated. Unfeasibility of the corresponding covariant description in gravitation for such an otherwise universally established concept as energy-momentum conservation could thus suggest inconsistency of the whole logical construction leading from the equivalence postulate to the theory of general relativity.

In contrast to other gravity theories of space-time curvature, in addition, general relativity is actually expected to be characterized by a so-called strong equivalence principle $[19,23,24$, $25,26]$. Consistent with the generalized principle of relativity and upheld by current theoretical and observational research $[23,24,26]$, however, this version of the equivalence principle still goes beyond what has originally been suggested by Einstein [21,25]. Were the theory of general relativity to arise directly from an independently postulated generalization of the principle of relativity, this theory might also have a covariant energy-momentum conservation law. It could then imply validity of the strong equivalence principle as well. Explanation of gravitation in terms of such related natural postulates as the generalized principle of relativity and covariant energy-momentum conservation would thus seem to be conceptually much more preferable compared to the combination of the former with a version of the equivalence principle.

Another mathematical issue has emerged in general relativity since a dynamical nature of the universe was derived from this theory by Friedmann [27] and observationally confirmed by Hubble $[28,29]$. When extrapolated backwards in time, such a dynamics of the universe has appeared to suggest the existence of a so-called cosmological singularity, which is associated with the initially infinite matter density $[12,15,17,19,29,30]$. Under certain restrictions on the motion of matter, a singular behavior of the Einstein field equations themselves has also been discovered later $[17,31,32]$, apart from what had been broadly noted prior to this by Einstein himself [33]. Such a behavior has then been generally established as well [34, 35, 36]. Typically viewed to be unacceptable for description of nature, singularities have thus still been admitted as a true physical element of general relativity under seemingly plausible assumptions [37].

In a recent work by the present author [38], however, a cosmological scenario rigorously avoiding the Friedmann initial singularity on account of a sufficiently general consideration of the equation of state for matter is reported. The present study is conceptually founded on such a resolvability of the other singularity problems as well. It utilizes both certain observational discoveries of several recent decades [29] and their general theoretical framework suggested in [38]. An interpretation of the theory of general relativity and its manifestations that does arise directly from the generalized principle of relativity and covariant energy-momentum conservation is thus exposed herein. In agreement with the strong equivalence between gravitation and inertia, this interpretation is not underlain by an equivalence postulate. The conservation law originally proposed by Levi-Civita [3] and Lorentz [4] is then specifically reexamined in terms of the suggested interpretation. Despite seemingly legitimate objections to that proposal $[11,13,14,17]$, it is eventually found to be principally consistent both with what is generally expected from energy-momentum conservation and with what is known about gravitation.

\section{Background}

The key outcome of the theory of general relativity is the Einstein field equations:

$$
G_{i j}=\kappa T_{i j} \Longleftrightarrow R_{i j}=\kappa\left(T_{i j}-\frac{1}{2} g_{i j} T\right) .
$$


Here $g_{i j}, R_{i j}$, and $G_{i j} \equiv R_{i j}-(1 / 2) g_{i j} R$ are the metric, Ricci, and Einstein tensors, respectively, $T_{i j}$ is the matter energy-momentum tensor, $i, j=0,1,2,3$ (as all Latin indices are assumed hereafter), $T \equiv T_{i}^{i}, R \equiv R_{i}^{i}(=-\kappa T)$ is the Ricci scalar curvature, the sign conventions used in [17] are adopted herein, and $\kappa=8 \pi G / c^{4}$ is the Einstein constant, where $G$ is the Newton constant and $c$ is the speed of light. As discussed in [38], the Einstein field equations without explicit identification of the so-called cosmological constant could generally be used when all phases of matter are allowed for. The vacuum phase of matter is thus also included (by $T_{i j}$ ) in Eqs. (1). Its existence has originally been postulated in [39].

In the absence of matter, $T_{i j}=0$, Eqs. (1) reduce to

$$
R_{i j}=0 .
$$

However, Eqs. (2) are still conventionally viewed as insufficient for the flatness of space-time. The latter property is identified by vanishing of the Riemann tensor $[12,13,15,17,18,19]$ :

$$
R_{k i l j}=0 .
$$

A nonvanishing space-time curvature when $R_{k i l j} \neq 0$ is thus expected to be principally sustainable even when the entire space-time is empty. Such a space-time curvature would have to imply a nonzero energy-momentum for the field of gravitation. The latter is then interpreted as the source of itself. Construed in terms of general relativity, our experience may also be viewed to suggest that the curvature is present when Eqs. (2) hold near a spatially compact gravity source. This could be seen $[14,17]$ as contradicting the interpretation of $G_{i j} / \kappa$ in Eqs. (1) as the gravitation energy-momentum tensor [3, 4]. Another argument in [14, 17] against this interpretation of $G_{i j} / \kappa$ is discussed in Sec. 5.1, whereas objections of a largely philosophical nature raised in this context by Einstein $[11,13]$ are considered in Sec. 5.2.

\section{Nonsingular general relativity}

\subsection{Generally nonsingular gravitational collapse}

From the present perspective, the main message of Ref. [38] is that a singularity resulting from the attractive gravitation of matter could be eliminated when temperature is also allowed for in the matter equation of state. In the Friedmann equations, in particular, the (large-scale) temperature happens to be implicitly present automatically. It is represented in terms of the scale factor. The singularity is thus avoided when the equation of state is generally permitted to vary with the latter parameter [38]. This is due to a (temperature-dependent) transition from matter to its vacuum phase [39] suggested by the Friedmann equations at the extreme temperatures characterizing a vicinity of the universe initial state. Such a phase transition circumvents the strong energy condition required by the singularity theorems [15, 34, 35, 36, 37].

Since the vacuum phase thus has to be a high-temperature origin for all the matter of a conventional phase existing in the universe [38], however, the above message could be consistently generalized to the Einstein field equations themselves. One would then have to anticipate an analogous phase-transition mechanism to become relevant at the extreme temperature conditions usually expected to characterize a vicinity of the final state of any matter gravitational collapse [40]. In particular, possibilities relevant to such a generalization in the context of black holes have been discussed in [41]. Although the Schwarzschild black hole singularity arises at a point, for example, it results from the gravitational collapse of a macroscopic object. It thus has to be precluded in the framework of such a macroscopic theory as general relativity.

The dependence of a matter equation of state on temperature still generally makes the number of unknowns in the Einstein field equations larger than the number of equations for the tensor components. In principle, however, this is expected to be remediable with an additional equation representing temperature dependence on the gravitationally caused matter motion. 


\subsection{Nonsingular empty space-time}

Under the strong energy condition [15, 34, 35, 36, 37], a singularity could however arise in an empty space-time as well $[37,42]$. The cosmological singularity when the space-time is empty has in fact been a major element of the corresponding theoretical research [30]. The mechanism of avoiding gravitational-collapse singularities discussed in Sec. 3.1 thus appears to be irrelevant. The empty space-time might then have to only stay flat to avoid its singularity [43, 44].

Generally constructible near a hypersurface of constant time $[12,15,17,19,30,32,36,37$, $45,46]$, the synchronous frame ought to be suitable for deriving invariant space-time properties. The empty space-time in such a frame thus indeed gives rise to a singularity $[17,30,32]$. This is seen from

$$
-R_{0}^{0}=\frac{1}{2} \frac{\partial \chi_{\alpha}^{\alpha}}{\partial t}+\frac{1}{4} \chi_{\alpha}^{\beta} \chi_{\beta}^{\alpha}=0
$$

where $\chi_{\alpha \beta} \equiv-\partial g_{\alpha \beta} / \partial t, g_{\alpha \beta}$ with $\alpha, \beta=1,2,3$ (as assumed for all Greek indices hereafter) is the spatial part of $g_{i j}, \chi_{\alpha}^{\beta} \equiv-g^{\beta \gamma} \chi_{\alpha \gamma}$, and $t$ is the time. Upon using [17] scalar inequality

$$
\chi_{\alpha}^{\beta} \chi_{\beta}^{\alpha} \geq \frac{1}{3}\left(\chi_{\alpha}^{\alpha}\right)^{2}
$$

which is obvious when $\chi_{\alpha}^{\beta}$ turns diagonal with the coordinate transformation, one obtains

$$
\frac{\partial \chi_{\alpha}^{\alpha}}{\partial t}+\frac{1}{6}\left(\chi_{\alpha}^{\alpha}\right)^{2} \leq 0 \Longleftrightarrow \frac{\partial}{\partial t}\left(\frac{1}{\chi_{\alpha}^{\alpha}}\right) \geq \frac{1}{6}
$$

When $\chi_{\alpha}^{\alpha} \neq 0, \chi_{\alpha}^{\alpha}=[\partial(-g) / \partial t] /(-g)=-\left[\partial\left(-g^{-1}\right) / \partial t\right] /\left(-g^{-1}\right)$ in inequality (6) thus turns infinite with either $g \equiv \operatorname{det}\left(g_{i j}\right)=\operatorname{det}\left(g_{\alpha \beta}\right)=0$ or $g^{-1}=\operatorname{det}\left(g^{i j}\right)=\operatorname{det}\left(g^{\alpha \beta}\right)=0$ within a finite time interval $[17,30,32]$. In particular, $[\partial(-g) / \partial t] /(-g) \rightarrow \pm \infty$ when either $g \rightarrow 0$ or $\partial(-g) / \partial t \rightarrow \pm \infty \Rightarrow g \rightarrow \infty \Leftrightarrow g^{-1} \rightarrow 0$. Likewise, $-\left[\partial\left(-g^{-1}\right) / \partial t\right] /\left(-g^{-1}\right) \rightarrow \pm \infty$ when either $g^{-1} \rightarrow 0$ or $\partial\left(-g^{-1}\right) / \partial t \rightarrow \mp \infty \Rightarrow g^{-1} \rightarrow \infty \Leftrightarrow g \rightarrow 0$.

The above behavior has still been commonly attributed only to such a property of synchronous coordinates as (caustic) intersection of their geodesically identified time lines [15, 17, $36,37]$. When the space-time is empty, however, such coordinates could not be rendered irrelevant by a matter pressure. Unrestrictedly constructible, they persist then as comoving $[17,47]$ up until the singularity is reached. The invariance of $g$ thus ought to suggest the existence of a universal physical feature of space-time itself underlying their singular behavior. (Focusing of hypersurface-orthogonal time-like geodesics in a curved empty space-time is in fact described invariantly by the Raychaudhuri equation as well [15, 31, 36, 37], although such a description also implies the relevance of synchronous coordinates.) This feature is discussed in Sec. 3.3.

In the synchronous frame, however, the singularity could be avoided only when $\chi_{\alpha}^{\alpha}=0$. Since $d l^{2}=-g_{\alpha \beta} d x^{\alpha} d x^{\beta}=-g^{\alpha \beta} d x_{\alpha} d x_{\beta}$ is then the square of a spatial interval, both $g_{\alpha \beta}$ and $g^{\alpha \beta}$ also have to be at least negative semidefinite. Without their singularities, when $g \neq 0$ and $g^{-1} \neq 0$, they could not have a zero eigenvalue and so are actually negative definite. Eq. (4) (with $\chi_{\alpha}^{\alpha}=0$ ) thus implies that all $\chi_{\alpha \beta}=\chi^{\alpha \beta}=0$, for their components then arise from those of a respective vector that has to be zero (for $g \neq 0$ and $g^{-1} \neq 0$ ).

(When space-time is filled with a perfect fluid whose energy-momentum tensor meets the strong energy condition, the above formalism still applies and leads to $T_{i j}=0$ [32]. This contradiction is avoided if the synchronous frame is rendered physically unrealizable by pressure gradients arising in the fluid $[17,48]$ and if the fluid phase transition to vacuum is allowed for.)

As also highlighted in $[17,32]$, however, the Ricci and Riemann tensors defined with $g_{\alpha \beta}$ on hypersurfaces of a constant time, $P_{\alpha \beta}$ and $P_{\gamma \alpha \sigma \beta}$, respectively, differ from their four-dimensional (4D) counterparts only by terms that vanish with $\chi_{\alpha \beta}=0$. Since $P_{\alpha \beta}=0 \Longleftrightarrow P_{\gamma \alpha \sigma \beta}=0$ generally holds, the existence of such a stationary reference frame as the synchronous one with $\chi_{\alpha \beta}=0$ is thus found to be sufficient for the empty space-time to be flat $[17,32,45,49]$. The above singularity is then eliminated only when there holds equivalence relation

$$
R_{i j}=0 \Longleftrightarrow R_{k i l j}=0
$$


whose underlying tensor equalities accentuate its validity independently of the reference frame. This relation is thus what ought to generally identify a nonsingular empty space-time. Since inequality (5) is key for arriving at (7), however, so is the nonlinearity of the Ricci tensor.

Strengthening [33], flatness is proved in [45] for a stationary space-time outside matter sources that becomes asymptotically flat faster than in the Newtonian gravity. As the outside flatness is suggested above to be generally enforceable, however, this proof in [45] is actually also eventually underlain by reduction of the stationary space-time to that in a synchronous frame. This reduction is permitted due to implications of the Hopf maximum-principle theorem.

Although test time-like trajectories arising from different points of a space-like hypersurface may intersect even in flat space-time, such an intersection has to come from their distinct initial directions. These intersecting trajectories do not thus actually qualify as time-like geodesics. Since such respective geodesics have to be parallel in flat space-time, their invariantly-described focusing has to suggest the presence of a Riemann curvature [50]. Also staying intact in terms of the comoving coordinates, such an irrotational focusing for empty space-time could not then be dismissed just as a failure of the synchronous frame $[15,17,36,37]$. In particular, its elimination above does formally lead to flat empty space-time with no focusing geodesics.

\subsection{Reinterpretation of relevant theoretical findings}

When no matter at all is present in the entire space-time, relation (7) simply suggests that the space-time singularity prohibits a gravitational energy-momentum to be the only source of itself. Since the boundary between matter and the nearby empty space-time is defined invariantly, however, the outcome of Sec. 3.2 above has to be applicable to a 4D region outside conventional sources of gravitation as well. The gravitational field could not then extend beyond the boundaries of its material sources with the ambient space-time.

Without the interpretation emphasized herein below, such a curious mathematical property has been discussed only for a lower-dimensional $(2+1)$ general relativity [51]. Its validity there is not underlain by a singularity of empty space-time. (In the $4 \mathrm{D}$ general relativity, an analogous piecewise flat model has then been suggested and discussed in [52]. In an admittedly curved empty space-time, concurrence of the 4D regions of nonzero energy-momentum for gravitation and matter has also been discussed [53] as a hypothesis questioning reality of the energy transfer by gravitational waves predicted for a binary pulsar [15, 24, 26, 54, 55].) Apart from the existence of gravitational waves $[9,12,15,17,18,19,24,26,42,44,54,55,56,57,58$, $59,60]$, the apparent curvature discontinuity at the source boundary in a real, 4D, space-time thus simply appears to perspicuously contradict the associated elementary experience.

In particular, the conclusions of Sec. 3.2 also have to apply to the space-time around a Schwarzschild mass $[12,13,15,17,18,19]$. A transitional process with which such a space-time reaches its (static) Schwarzschild metric would indeed be inevitable when one arrow of time is dominant. The Birkhoff theorem $[12,17,18,19,35,61]$ then also suggests that such a transition has to be spherically asymmetric. It ought to typically result from asymmetric initial conditions. These could arise when a spherically symmetric source has asymmetric surroundings or forms out of an asymmetric origin, as say in the recent observations of gravitational waves [59]. In a synchronous frame, however, such a transitional process would have to formally take an infinite time. Since the singularity then arises within a finite time, the outside space-time flatness is thus enforced despite the mathematical existence of the nonflat static metric.

The conclusions of Sec. 3.2 have to apply even to a hypothetical situation when a finite Schwarzschild mass alone grows out of nothing while its spherical symmetry is ever maintained. The Birkhoff theorem then still implies that this process could give rise to the (static) Schwarzschild metric only with a spherically asymmetric transition. When the ambient spacetime is empty, however, there is literally no perturbation from which such a transient asymmetry could arise. When this space-time is assumed to be originally flat, therefore, it would have to remain such even after the (symmetric) formation of a finite Schwarzschild source. 
In special relativity, however, the Lorentz transformation of space and time intervals with respect to a moving inertial frame applies only when such intervals are materially defined. For the extended range of all reference frames in general relativity, the concept of local space-time curvature thus also ought to be physically meaningful only within a macroscopically continuous (in space and time) medium whose background presence would be implied [62, 63]. The above seemingly paradoxical inferences would then have to be attributed to the absence of such a medium in a truly empty space-time region. Matter is thus viewable as both a source of space-time curvature and a facility due to which such a curvature acquires a physical definition.

From a purely mathematical perspective, one can still formally obtain nonflat exact solutions of the Einstein field equations for an empty space-time region. Apart from the Schwarzschild metric, for example, such solutions have been extensively discussed in the past in the context of gravitational waves $[18,42,44,55,56,58]$. To be principally measurable, however, these solutions ought to imply the existence of a medium due to which their nonflat metrics could actually be manifested. It is the requirement of physical meaningfulness for mathematical variables, suggested by the singularity of empty space-time, that generally eliminates what might be seen as a contradiction between the mathematical existence of nonflat solutions for empty space-time and the respective space-time flatness inferred in Sec. 3.2.

In Sec. 3.2, the singularity highlighting what has just been emphasized arises from nonlinearity of the Einstein field equations. Singularities in whose context reality of gravitational waves has repeatedly been discussed were also found to come from nonlinearities of these equations $[18,42,43,44,55,56]$. The issue of physical identification for space-time curvature is still more general and fundamental than a specific cause thus suggested for any of such singularities. Independent of the frame of reference, this issue implies no privilege for the synchronous frame either. Due to the physical nature of its construction, however, such a frame relates the singularity of an empty space-time region to the flatness of this region in terms of the respective invariant descriptions. Always constructible and stably comoving in such an empty region $[17,47]$, it thus happens to be endowed with the capacity of exposing how space-time flatness generally results from the lack of physical meaning for the respective curvature.

Within matter, in particular, the focusing of geodesics arising orthogonally from a space-like hypersurface is physically meaningful. When pressure gradients form with the radiation phase, however, it ceases to apply to matter motion [17, 48]. Under yet certain conditions $[15,34,35$, 36, 37], as suggested in Sec. 3.1, transition to the vacuum phase of matter would also prevent the singularity of gravitational collapse for a conventional matter phase. If such geodesics are still theoretically constructed in a curved empty space-time, the resulting singularity is thus made unavoidable. This contradiction signals the lack of physical meaning for the space-time curvature in such an empty region. Forced to be invariantly flat once the above geodesics have given rise to a synchronous frame, the nonsingular empty space-time then renders the geodesics parallel to avoid their focusing. This also eliminates the contradiction. Generally definable with such geodesics, the synchronous frame thus has just a natural physical mechanism of generating the singular behavior and thereby enforcing space-time flatness exactly when the respective curvature would fundamentally have to be physically unrealizable in any frame.

\subsection{Manifestation of the gravitation phenomenon}

Even bar the mathematical inferences in Sec. 3.2, therefore, the entirely empty space-time still has to be flat due to the lack of physical meaning for space-time curvature without matter. The empty space-time area outside a material source of gravitation also has no physically identified local curvature to be related to that within the source itself. Manifested in this failure of space-time curvature to extend beyond such a gravity source, a curvature discontinuity thus has to arise at the source boundary. Whether in our experience or ever since the very beginning of the universe, however, gravitation seems to have never been such as would observationally or theoretically be required to take place in a truly empty space-time region. 
What ought to have ever filled space-time to define its curvature is a medium arising from vacuum decay in the universe [38]. This medium then has to consist of the decaying vacuum itself and a macroscopically continuous product of the decay. Thus required to have equal amounts of matter and antimatter and a nonzero pressure, such a product whose existence is already established is Cosmic Microwave Background Radiation (CMBR) [40]. CMBR then defines space intervals. Time intervals are thus identified by such interrelated processes as cosmic-vacuum decay and the vacuum-driven CMBR spatial expansion. Characterized by increasing entropy [64], these processes are representative of the second law of thermodynamics.

Expansion of the spatially flat universe [38] would have to continue to infinite values of the scale factor [65]. At least one of the above components, CMBR, should still be present in such a universe till the very end of the expansion process. Having a locally negligible gravitational density, as in the present universe, the combination of CMBR with decaying vacuum at the final stages of vacuum decay would then be ideally suitable for the role of the background medium. In particular, such a medium would not require a discernible quantitative modification of any of the predictions of general relativity formally obtained for the truly empty space-time region when the singularity there (discussed in Sec. 3.2) is ignored. By eliminating that singularity of empty space-time, however, this medium permits the space-time filled with it to actually curve according to such predictions for other (more substantial) energy-momentum sources. As suggested in Sec. 3.1, its possible transition from CMBR to the vacuum phase also allows to avoid a singularity of the medium gravitational collapse. Such a phase transition could then arrest a gravitational collapse of the other matter sources of space-time curvature as well.

The macroscopic nature of general relativity is thus essential for the gravity manifestations described by this theory. What is viewed as a point source, therefore, actually has to be itself a macroscopic object with the own internal structure, although it is often described on a yet larger macroscopic scale as a $\delta$-function. Gravitation thus ought to be absent in ideally microscopic processes $[66,67]$, for no medium exists in such processes to define curvature of the ambient space-time. Identified by the background medium, gravitational waves then have to be physically analogous to waves of a hydrodynamic nature rather than to electromagnetic radiation.

As an "ether" for defining and transmitting space-time curvature [63], the background medium could thus continuously extend the gravitational field beyond the main spatially compact field sources. This is additionally discussed in Sec. 5.1. Despite the apparent contradiction outlined in the end of Sec. 2, therefore, the outcome of Sec. 3.2 might imply that $G_{i j} / \kappa$ in Eqs. (1) is indeed the energy-momentum tensor for gravitation $[3,4]$. In the presence of background medium, such a view would not disagree with our experience. Standardly rederived in Sec. 4.2, this energy-momentum tensor is then discussed in Sec. 5. Before the derivation, however, gravitation is discussed in Sec. 4.1 in terms of covariance of the total energy-momentum conservation, which is thus interpreted to underlie this phenomenon in general relativity.

\section{Generalized energy-momentum conservation}

\subsection{The principle of relativity and energy-momentum conservation}

As mentioned above, the absence of a cosmological term other than that arising from a decaying vacuum phase in the matter energy-momentum tensor is assumed in Eqs. (1). This is suggested by the scenario of universe evolution derived in [38]. Without matter, Eqs. (1) are thus satisfied by flat space-time, which is also their only nonsingular solution then.

Let us consider a material subsystem that is a part of such a larger total system as has a background medium around the subsystem. Capable of transmitting space-time curvature, this medium by itself is assumed to have negligible effects on such a curvature. Such an overall finite system could stand for a spatially compact matter source filled with the background medium around one or more of its smaller subsources in an otherwise flat empty space-time. 
The system could however also cover an open infinite space-time with say a spatially compact gravity source surrounded by the background medium with or without other such sources [68].

In an inertial frame without gravitation, energy-momentum conservation for the above subsystem is due to the homogeneity of its flat space-time [16, 69, 70]. A subsystem like this could arise say from such a small energy density [71] of matter as just defines a reference frame when other system gravity sources are absent. As also the background medium of the overall system, such a subsystem would thus have a negligible gravitational effect on spacetime curvature while being relevant to nongravitational interactions. Upon its transition to a noninertial frame, however, the space-time homogeneity is lost. In particular, this is illustrated by $v^{2} / c^{2}$ ( $v$ is the subsystem relative velocity) in the Lorentz transform of space-time intervals. Defined by matter alone, this magnitude would cease being constant in a noninertial frame.

However, the transition to a noninertial frame is meaningful only when there exists an external material source within the overall system due to which the frame noninertiality could be defined [72]. This transition is also accomplishable only on account of an additional energymomentum gradient associated with such a source. Unlike a constant energy-momentum shift for switching between two inertial frames, therefore, this additional gradient inevitably becomes a part of the differential balance for energy-momentum conservation in the system. It would thus have to be allowed for by formulation of the generalized principle of relativity, i.e. when the principle of relativity applied to inertial reference frames is extended to noninertial ones.

When the nature of frame noninertiality is due to a conventionally nongravitational interaction, the system energy-momentum conservation is admittedly maintained. The energymomentum gradient associated with the space-time inhomogeneity affecting the subsystem is then offset by the gradient of energy-momentum for the external matter by interaction with which the subsystem noninertiality arises. The rate of energy-momentum change characterizing this interaction with the subsystem could thus in principle be of any magnitude. (In particular, such an energy-momentum conservation is often viewed as the third law of Newton.)

In terms of energy-momentum conservation for the system as a whole, however, noninertiality of the subsystem is actually permitted even without nongravitational interactions. In particular, such a subsystem noninertial motion still has to be defined by the existence of an outside matter source. The energy-momentum gradient for the underlying (noninertial) space-time curvature could then be offset merely by the gradient of material energy-momentum arising from this external source. Although the background medium defines a relatively noninertial space-time curvature, it could not be the matter source responsible for energy-momentum balance in the presence of this curvature. Such an energy-momentum source thus has to be an element of the total system other than the background medium surrounding the subsystem.

Due to its very presence, therefore, an outside source permits the existence of a noninertial frame for the subsystem at the expense of the matter energy-momentum gradients generated by this source [72]. Such a reference frame thus ought to be allowed for by a frame-independent description. Once the subsystem density [71] has ceased being gravitationally negligible, however, its energy-momentum would likewise determine the frame space-time curvature specifying the relative acceleration of the outside matter [73]. This attributes gravitation to such a relative noninertial space-time curvature as results from the very existence of a conventionally noninteracting matter, by which the noninertiality is defined, within a material system and the overall energy-momentum conservation in this system being maintained for any reference frame.

Gravitation is thus interpretable in terms of generalization of the concept of energymomentum conservation to noninertial reference frames. This concept is particularly relevant when substantial energy sources in a matter system to which it is applied are connected only by a gravitationally negligible medium that allows their noninertial space-time curvature to form with respect to each other. Since such a curvature is then defined by a matter-generated frame, as suggested by the generalized principle of relativity, its matter-related dynamics ought to be described invariantly. Obtained by variation of the total action in the components of the metric tensor, this description is due to the Einstein field equations $[12,13,15,17,18,19]$. 
The (generalized) principle of relativity is thus a fundamental postulate, as in the special relativity theory for inertial frames. Consistent with the strong equivalence principle $[19,23,24$, $25,26]$, as suggested by the above discussion in terms of inertial effects alone, this interpretation does not need such a principle as a separate postulate. Since the equivalence postulate is often viewed as contradicting the localizability of gravitational energy-momentum [12, 16], however, elimination of this postulate in the presence of inertial-frame flat space-time background [15] has to permit the local energy-momentum conservation to be covariantly maintained.

\subsection{Reinterpretation of standard formalism}

Describing interaction between two matter fields arising together from the flat background of empty space-time, Eqs. (1) thus have to covariantly stand for the total energy-momentum conservation. That flat space-time is seen from Sec. 3.2 to be the only nonsingular solution of these equations without matter is then a posteriori consistent with such interpretation of Eqs. (1). In this nonsingular framework, the metric tensor also defines a purely dynamical variable [15]. In terms of such a variable, space-time curvature underlying the energy-momentum for gravitation is identified as coming from frame noninertialities within the respective macroscopically continuous matter system. The formalism giving rise to the first Noether theorem $[16,69,70]$ then has to lead to a proper conservation law that is consistent with Eqs. (1). It still applies solely when the matter and gravitational field actions are varied together, as is the only nonsingular option for them to nontrivially exist. Such total action is thus $[12,13,15,17,18,19]$ :

$$
S_{T}=-\frac{1}{2 c \kappa} \int_{\Omega} R \sqrt{-g} d \Omega+\frac{1}{c} \int_{\Omega} \Lambda \sqrt{-g} d \Omega,
$$

where $R$ and $\Lambda$ are the Lagrangian densities for the mutually interacting material fields of noninertial space-time curvature and matter itself, respectively. Filled with matter, $\Omega$ is then the curved integration area. It has coordinates $x^{i}$ and boundary $\partial \Omega$.

In the present context, $S_{T}$ in Eq. (8) is invariant to infinitesimal translations of flat spacetime coordinates $z^{i}$. The latter form four-vector $z$. Let $S_{T}$ thus undergo the variation under such translations $d z^{i} \equiv \epsilon \delta z^{i}$ for an infinitesimal $\epsilon$. Coordinates $x^{i}$ of their four-vector $x$ then transform into the coordinates $x^{\prime i}=x^{i}+\epsilon \xi^{i}+o\left(\epsilon \delta z^{i}\right)$ of four-vector $x^{\prime}$, where $\xi^{i} \equiv\left(\partial x^{i} / \partial z^{j}\right) \delta z^{j}$ are the components of four-vector $\xi$. Such a coordinate translation is still identifiable by the permitted coordinate transformation. For the contravariant metric tensor, this implies [74]:

$$
g^{\prime i j}\left(x^{\prime}\right)=g^{\prime i j}[x+\epsilon \xi+o(\epsilon \delta z)]=g^{k l}(x) \frac{\partial x^{\prime i}}{\partial x^{k}} \frac{\partial x^{\prime j}}{\partial x^{l}}=g^{i j}(x)+\epsilon\left(g^{i l} \xi_{, l}^{j}+g^{j k} \xi_{, k}^{i}\right)+o(\epsilon \delta z) .
$$

Since matter is absent and space-time is flat beyond $\Omega$, however, the integrands in Eq. (8) vanish there. Arising when $S_{T}$ is varied, the integrals of divergence for their products with $\xi$ are therefore eliminated although $\xi_{\mid \partial \Omega}\left(=\delta z_{\mid \partial \Omega}\right) \neq 0$ is relevant to assume [75]. At the order of $\epsilon$, the variation of either integral in Eq. (8) resulting from the above translations is then reduced to the integral of ordinary variation for the respective integrand alone [13]. Identical to the Lie derivative $[12,15,19]$ at this order, $£_{-\xi} g^{i j}(x)$, such a variation of $g^{i j}(x)$ thus becomes:

$$
\delta g^{i j}(x)=g^{\prime i j}(x)-g^{i j}(x)=\epsilon\left(-\xi^{k} g_{, k}^{i j}+g^{i k} \xi_{, k}^{j}+g^{j k} \xi_{, k}^{i}\right)+o(\epsilon \delta z)=\epsilon\left(\xi^{i ; j}+\xi^{j ; i}\right)+o(\epsilon \delta z) .
$$

Applying to $S_{T}$ in Eq. (8) the formalism typically used (say in $[17,19]$ ) in the context of scalar invariance of the matter action alone, one could obtain from the invariance of $S_{T}$ to the above coordinate translations at the flat empty background (when space-time is flat outside $\Omega$ [75]) that

$$
\int_{\Omega}\left(G_{i}^{j}-\kappa T_{i}^{j}\right)_{; j} \xi^{i} \sqrt{-g} d \Omega=0
$$

Consistently with its definition in Eqs. (1), the Einstein tensor arises here as

$$
G_{i j}=\frac{1}{\sqrt{-g}}\left[\frac{\partial\left(R^{*} \sqrt{-g}\right)}{\partial g^{i j}}-\frac{\partial}{\partial x^{k}} \frac{\partial\left(R^{*} \sqrt{-g}\right)}{\partial g_{, k}^{i j}}\right]
$$


where $R^{*}$ is defined in terms of Christoffel symbols $\Gamma_{i j}^{k}$ as

$$
R^{*} \equiv g^{i j}\left(\Gamma_{i l}^{k} \Gamma_{j k}^{l}-\Gamma_{i j}^{l} \Gamma_{l k}^{k}\right)
$$

$R^{*}$ thus stands for the terms in $R$ with only first derivatives of $g_{i j}$, whereas the validity of $\sqrt{-g}\left(g^{i k} \delta \Gamma_{i k}^{l}-g^{i l} \delta \Gamma_{i k}^{k}\right)=0$ for the flat space-time beyond $\Omega$ naturally eliminates such terms with the second derivatives [76]. The matter energy-momentum tensor is then defined as

$$
T_{i j}=\frac{2}{\sqrt{-} g}\left[\frac{\partial(\Lambda \sqrt{-g})}{\partial g^{i j}}-\frac{\partial}{\partial x^{k}} \frac{\partial(\Lambda \sqrt{-g})}{\partial g_{, k}^{i j}}\right] .
$$

Since $\delta z \neq 0$ could be arbitrary at any $z, \xi^{i}$ are also viewable as arbitrary. Eq. (11) thus leads to

$$
\left(G_{i}^{j}-\kappa T_{i}^{j}\right)_{; j}=0
$$

Due to the contracted differential Bianchi identities, in particular, Eqs. (15) also imply

$$
T_{i ; j}^{j}=0
$$

Irrespective of their status for the conservation law, Eqs. (1) still arise with matter as describing dynamics of the material field of relative space-time curvatures from the extremum of $S_{T}$. The latter is then varied in the metric tensor components as these (and their Christoffel symbols) are fixed beyond $\Omega$ by the space-time flatness there. When uniquely derived from both Eqs. (1) and (15), however,

$$
\left(G_{i}^{j}-\kappa T_{i}^{j}\right)_{, j}=0
$$

are attributable to the background space-time flatness. Eqs. (17) thus covariantly express the total energy-momentum conservation underlying Eqs. (1) as well. $G_{i}^{j} / \kappa$ is then also what is viewable as the energy-momentum tensor for gravitation $[3,4,77,78]$, i.e. for the tensor field of materially defined space-time curvatures arising from the respective noninertial frames.

\section{Summary and further discussion}

\subsection{The covariant conservation law in theory and observations}

Viewed as a property of empty space-time, energy-momentum of the gravitational field has been typically sought to be such as would turn Eqs. (16) into an ordinary divergence [16, 18]. Failing to yield a unique tensorial representation, this has given rise to a multitude of pseudotensors $[16,17,18,19,20]$. Identified up to an arbitrary term with vanishing ordinary divergence $[16,18]$, the suggested pseudotensors have thus largely originated from the so-called canonical expression for energy-momentum tensors in flat space-time $[2,18]$. Arising then from the scalar invariance of the matter action, however, the matter energy-momentum tensor defined by Eqs. (14) still generalizes such an expression to allow for the available space-time curvature. When described by $G_{i j} / \kappa$, where $G_{i j}$ is given by Eqs. (12) [consistently with its definition in Eqs. (1)], the gravitation energy-momentum is endowed with both a tensorial nature and the same form as for its matter counterpart in Eqs. (14). This joint form arises since a nontrivial existence is nonsingularly permitted only for the total action, such as is entirely defined with matter.

First suggested in $[3,4]$, however, $G_{i j} / \kappa$ is so implied by general relativity as the energymomentum for gravitation only because an empty space-time region nonsingularly described by this theory is thus rendered flat. Relation (7) for such a region is then found to be irrelevant to our observational experience since the actual space-time between spatially compact gravity sources is filled with a macroscopically continuous medium whose local gravitational effects ought to be negligible. Eliminating the singularity of empty space-time, this medium arises from vacuum decay in the universe. It thus also prevents a singularity of the own gravitational 
collapse. It is due to such a background medium that space-time curvature extends beyond its main sources consistently with the materiality of the Lorentz transform for inertial frames.

Interpreted as proportional to the energy-momentum tensor for the gravitational field, $G_{i j}$ thus has to describe transmission of the field space-time curvature. This phenomenon would be generated by transients adjusting the gravitational field to matter dynamics and phase transitions. Physically identified by the background medium, small-amplitude counterparts of these transients could be viewed as gravitational waves $[9,12,15,17,18,19,24,26,42,44,54$, $55,56,57,58,59,60]$. Responsible for such a proliferation of space-time curvature, the Einstein field equations with the background medium around all main gravity sources then describe the total energy-momentum conservation for matter consistently with known observations.

In the presence of a background space-time curvature, in particular, the energy-momentum of (relatively short) gravitational waves is typically approximated by an averaged second-order perturbation of the Einstein tensor $[12,17,60]$. This perturbation then represents what is relevant in the Einstein tensor itself when observed decay in the orbital period of a binary pulsar is also inferred from the radiated energy thus described far from the source [15, 24, 26, $54,55]$, where a deviation of the background curvature from flatness is neglected. The above medium in terms of which space-time curvature and gravitational waves themselves ought to be defined is then implied as negligible for such a background curvature as well. Agreement with the observations achieved under this (quadrupole) approximation would therefore have to be unaffected by the nature of gravitational waves and energy-momentum suggested herein.

When gravitation is treated to be a feature of empty space-time, $G_{i j} / \kappa$ is also typically disqualified [14, 17] as its energy-momentum tensor for being thus defined with Eqs. (12) conceptually differently from the symmetric $T_{i j}$ in Eqs. (14) (say in $[17,19]$ ). Although such $T_{i j}$ results from the variation of its respective action in the gravitational field components as well, unlike for $G_{i j}$ in Eqs. (12), such field coordinates are then viewable as external to the matter. From the present perspective, however, a nontrivial action for the gravitational field cannot exist alone. Actually defined only in terms of the matter field, it is just a constituent of the total action for matter. The total energy-momentum tensor thus arises from the totalaction invariance specified by the flat empty background rather than by the scalar nature of the action constituents. Differentiation with respect to the metric-tensor components and their first derivatives is then just an intermediate stage in such a procedure as has to formally define the total energy-momentum tensor, in which $G_{i j} / \kappa$ merely stands for the gravitational part.

Minimizing the total action, Eqs. (1) thus arise with matter and its frames [72] as the Euler-Lagrange equations $[15,18]$ for the materially defined field of relative space-time curvatures. As discussed say in $[17,19]$ and thereby implied in Sec. 4.2, when Eqs. (15) are derived, the matter equations of motion in such a prescribed field of gravitation are taken into account as well. [The equations of matter motion are also derivable from Eqs. (16) and thus from Eqs. (1) themselves [12, 57].] As generally expected, Eqs. (17) are then based both on the invariance of total action to symmetries of the flat empty background and on the equations of motion for matter [70]. Although their derivation from Eqs. (15) is also based on Eqs. (1), the material Euler-Lagrange dynamics of gravitation in Eqs. (1) actually expresses the same total energy-momentum conservation for the matter fields as a whole as Eqs. (17) themselves.

\subsection{Thermodynamics and matter creation}

Even when viewed to imply the existence of a curved empty space-time, the Einstein field equations have been repeatedly suspected and examined in terms of having a thermodynamic identity as their fundamental origin [79]. Such interpretations have still largely come from phenomenological extrapolations of the black hole thermodynamics [80]. When space-time curvature is defined only by matter itself and viewed as a relative measure of energy-momentum, however, this underlying fundamental identity is found to be the covariant formulation of total energy-momentum conservation for the respective macroscopically continuous material system. 
Raised by Einstein $[11,13]$, a conceptual objection to such an interpretation $[3,4]$ of his equations might stem from treating gravitation as an attribute of empty space-time. The zero total energy maintained by Eqs. (1) irrespective of the existence of matter thus seemed to disallow an energy-related mechanism for creation of the material world out of nothing. When Eqs. (1) are viewed to be entirely of a material nature, however, a phase of matter has to always persist in the universe described by them [38]. The mechanism for creation of conventional matter phases then also results naturally from general relativity. Like attractive gravitation [81], it is manifested consistently with the second law of thermodynamics. As initially noted by Levi-Civita [3, 11], this mechanism is due to Eqs. (1) and the contracted differential Bianchi identities, from which Eqs. (16) arise. It is represented by decay of the (high-temperature) vacuum phase of matter and creation of (low-temperature) conventional matter phases when Eqs. (1) [and (16)] are nonsingularly applied to the universe [27, 38]. Although such processes were hardly conceivable when energy-momentum conservation was discussed by T. Levi-Civita and A. Einstein [11], their thermodynamic origin thus comes from that of Eqs. (1) themselves.

When the Friedmann scale factor $a=0$, in particular, the vacuum-universe energy is zero [82], despite its nonzero energy density. (As discussed in [82], this is due to the lack of definition for a nonzero vacuum momentum and the relativistic invariance of the energy-momentum vector.) A constant part of the vacuum density then has to be offset by the corresponding term permitted in the left-hand side of the Einstein field equations. This generalized interpretation in [38] is currently viewable as a consequence of the total energy-momentum conservation. It is also attributable to the absence of noninertial frames with respect to the vacuum of a constant density. Manifestation of the second law of thermodynamics, decay of the vacuum phase of matter into a conventional matter phase thus proceeds in compliance with the first law as well.

As broadly discussed in [38], the creation of matter (and of the respectively implied antimatter) is then driven by the overall thermodynamic energy balance for vacuum. Like the inertial energy in attractive gravitation, this vacuum energy arises with matter when $a$ increases from 0 . Its conservation gives rise to vacuum decay into a new matter, which is meaningful due to the matter parts already created and relatively accelerated by the decaying phase. The vacuum density is thus decreased to offset the enhancement of the overall vacuum energy by the expansion work. When the density and pressure of the created matter are also (invariantly) allowed for, such work is done against the vacuum pressure and matter attractive gravitation by the repulsive vacuum gravitation and radiation pressure while the latter two act on the created matter. Described by the Friedmann equations, these processes are thus suggested by the Einstein field equations themselves. Manifested in terms of the above scale factor, the effect of temperature on the matter equation of state still has to be allowed for.

\section{Conclusions}

Conceptually extending the formalism applied to the Friedmann equations in [38], a singularity resulting from the attractive gravitation of matter is assumed here to be generally avoidable. This assumption implies that temperature-dependent phase transitions changing the equation of state would fundamentally have to be ubiquitously anticipated at the extreme conditions engendered by a matter gravitational collapse. The vacuum phase of matter thus formed would then prevent such a singularity. Since a curved empty space-time region is also recognized here as invariantly singular, however, implications of avoiding this type of singularity are examined as well. These are found to involve foundations of general relativity and existence of a covariant formulation for energy-momentum conservation in this theory. They also suggest a reinterpretation of the very nature of gravitation.

Due to nonlinear features of the Ricci tensor in a synchronous frame, the singularity of empty space-time is generally avoided only when the space-time in the empty region is invariantly flat. Inevitable focusing of geodesics arising orthogonally from a space-like hypersurface 
in such a curved space-time is thus suggested to be a property of space-time itself. Consistently with the potential of matter to undergo phase transitions and thereby avoid its gravitational collapse, this singular character of empty space-time is attributed to the lack of physical meaning for space-time curvature there. Relevance of such interpretation is particularly pronounced when the curvature is viewed to be compatible with the Lorentz transform. The latter is admittedly defined by matter alone. The synchronous frame is then also highlighted to generate an invariantly singular comoving behavior of its time lines and thus to enforce space-time flatness where the respective curvature could fundamentally have no physical identification.

Consistently with the observational experience, space-time curvature is still extendible beyond its spatially compact sources when the space-time outside them is filled with a macroscopically continuous medium. It is due to such a background medium that the variation of space and time intervals is definable. Having locally negligible gravitational effects, the most obvious real constituents of this medium could be the decaying cosmic vacuum and such a product of its disintegration as CMBR at the final stage of vacuum decay. Matter and its vacuum-phase decay thus comprise what endows space-time curvature with the physical meaning.

Based on the above understanding, the generalized principle of relativity is postulated and highlighted to imply the extension of energy-momentum conservation to noninertial reference frames. This postulate then also suggests direct identification of gravitation with inertia. Consistent with the strongest version of the equivalence principle, it does not thus require such a principle to underlie the theory of general relativity as a separate postulate. The equivalence postulate is also found to be inadequate in equating the relative space-time curvature (defined by matter) in conventional interactions to that of an empty space-time region, where no curvature has a material realization. Gravitation then arises when energy-momentum conservation is covariantly applied to a matter system whose different parts are connected at least by a background medium. This medium is what also defines the space-time curvature affecting the system parts in their relatively noninertial frames. Such frames are permitted on account of the energy-momentum gradients created by the matter sources to which the frames are identified.

In the context of the outlined macroscopic picture of gravity manifestation, gravitation ought to be irrelevant to solely microscopic interactions. Having only such a macroscopic origin as the vacuum phase of matter, whose decay also gives rise to the background medium, repulsive gravitation is still expected to link the microscopic creation of matter and antimatter with either respective thermodynamics. Consistently with the first and second [81] laws of thermodynamics in attractive gravitation, this link is manifested in terms of the energy conservation and entropy increase for the vacuum-phase decay. Macroscopically separated, two opposite cosmological time arrows then arise consistently with the respective thermodynamics of matter and antimatter from the microscopic CPT symmetry [38, 83].

Obtained from minimization of the total action for matter to the components of the metric tensor, the Einstein field equations are thus what invariantly describes the relative space-time curvature engendered by the field of matter and its noninertial frames. With such equations of motion for this curvature and their flat empty background, the differential energy-momentum balance is derivable as underlying these equations from the background invariance of the total action to infinitesimal coordinate translations. The Einstein tensor then has to stand for the energy-momentum of the gravitational field. Its small-amplitude approximation also has to describe what could be viewed as gravitational waves. Defined in terms of the background medium, their underlying nonlinear curvature transients ought to result from matter dynamics and phase transitions. The conceptual issue raised by Einstein $[11,13]$ in the context of such an original interpretation of his field equations $[3,4]$ is also eliminated with the entirely material nature of these equations and the cosmological scenario of vacuum decay derived in [38]. The absence of a constant part of the cosmological term suggested in [38] from this scenario is then interpretable as a particular manifestation of the total energy-momentum conservation as well. Gravitation is thus fully described in terms of covariant energy-momentum conservation for such a macroscopically continuous material system with no proper motion as the universe. 


\section{References}

[1] A. Einstein, Annln. Phys. 354, 769-822 (1916); also in: The Principle of Relativity: A Collection of Original Papers on The Special and General Theory of Relativity. Translated by W. Perrett, G. B. Jeffery, pp. 109-164 (Dover Publications, New York 1952).

[2] A. Einstein, König. Preuss. Akad. Wiss. (Berlin). Sitz., 1111-1116 (1916); also in: The Principle of Relativity: A Collection of Original Papers on The Special and General Theory of Relativity. Translated by W. Perrett, G. B. Jeffery, pp. 165-173 (Dover Publications, New York 1952).

[3] T. Levi-Civita, Rendiconti della Reale Accademia dei Lincei 26, 381-391 (1917); English translation and foreword by S. Antoci, A. Loinger, arXiv:physics/9906004 (1999).

[4] H. A. Lorentz, Proc. Kon. Akad. Wet. 20, 2-19 (1918);

H. A. Lorentz, Proc. Kon. Akad. Wet. 20, 20-34 (1918).

[5] E. Schrödinger, Physik. Zeit. 19, 4-7 (1918).

[6] H. Bauer, Physik. Zeit. 19, 163-166 (1918).

[7] G. Gorelik, In: Einstein Studies in Russia. Einstein Studies 10. Edited by Y. Balashov, V. Vizgin, pp. 17-43 (The Center for Einstein Studies 2002) and references therein.

[8] A. Einstein, Physik. Zeit. 19, 115-116 (1918).

[9] A. Einstein, König. Preuss. Akad. Wiss. (Berlin). Sitz., 154-167 (1918);

A. S. Eddington, Proc. R. Soc. Lond. Ser. A 102, 268-282 (1922).

[10] A. Einstein, König. Preuss. Akad. Wiss. (Berlin). Sitz., 448-459 (1918).

[11] C. Cattani, M. De Maria, In: The Attraction of Gravitation: New Studies in the History of General Relativity. Edited by J. Earman, M. Janssen, J. D. Norton, pp. 63-87 (The Center for Einstein Studies 1993).

[12] C. W. Misner, K. S. Thorne, J. A. Wheeler, Gravitation. W. H. Freeman and Company, San Francisco (1973).

[13] W. Pauli, Theory of Relativity. Pergamon Press (1958).

[14] L. D. Faddeev, Sov. Phys. Usp. 25, 130-142 (1982).

[15] R. M. Wald, General Relativity. The University of Chicago Press, Chicago and London (1984).

[16] A. Trautman, In: Gravitation: An Introduction to Current Research. Edited by L. Witten, pp. 169-198 (John Wiley \& Sons 1962) and references therein.

[17] L. D. Landau, E. M. Lifshitz, The Classical Theory of Fields, Fizmatlit, Moscow (2003) (in Russian).

[18] J. Weber, General Relativity and Gravitational Waves. Interscience Publishers, New York (1961) and references therein.

[19] S. Weinberg, Gravitation and Cosmology. John Wiley \& Sons (1972).

[20] L. B. Szabados, Liv. Rev. Rel. 12, 4 (2009) and references therein. 
[21] A. Einstein, J. Rad. Elektr. 4, 411-462 (1907); J. Rad. Elektr. 5, 98-99 (1908);

A. Einstein, Annln. Phys. 340, 898-908 (1911); also in: The Principle of Relativity: A Collection of Original Papers on The Special and General Theory of Relativity. Translated by W. Perrett, G. B. Jeffery, pp. 97-108 (Dover Publications, New York 1952).

[22] T. Damour, Class. Quant. Grav. 13, A33-A41 (1996);

T. Damour, C. R. Acad. Sci. Paris Ser. IV 2, 1249-1256 (2001).

[23] T. Damour, In: 300 Years of Gravitation. Edited by S. W. Hawking, W. Israel, pp. 128-198 (Cambridge University Press 1987) and references therein.

[24] C. M. Will, Theory and Experiment in Gravitational Physics. Cambridge University Press (1993) and references therein.

[25] E. Di Casola, S. Liberati, S. Sonego, Am. J. Phys. 83, 39-46 (2015) and references therein.

[26] I. H. Stairs, Liv. Rev. Rel. 6, 5 (2003) and references therein;

T. Futamase, Y. Itoh, Liv. Rev. Rel. 10, 2 (2007) and references therein;

C. M. Will, Proc. Nat. Acad. Sci. USA 108, 5938-5945 (2011) and references therein;

C. M. Will, Liv. Rev. Rel. 17, 4 (2014) and references therein.

[27] A. Friedmann, Z. Phys. 10, 377-386 (1922) [Gen. Rel. Grav. 31, 1991-2000 (1999)];

A. Friedmann, Z. Phys. 21, 326-332 (1924) [Gen. Rel. Grav. 31, 2001-2008 (1999)].

[28] E. Hubble, Proc. Nat. Acad. Sci. USA 15, 168-173 (1929).

[29] S. Weinberg, Cosmology. Oxford University Press, New York (2008).

[30] V. A. Belinski, Int. J. Mod. Phys. D 23, 1430016 (2014) and references therein.

[31] A. Raychaudhuri, Phys. Rev. 98, 1123-1126 (1955);

A. Raychaudhuri, Phys. Rev. 106, 172-173 (1957).

[32] A. Komar, Phys. Rev. 104, 544-546 (1956).

[33] A. Einstein, Rev. Univ. Nac. Tucuman Ser. A 2, 11-15 (1941);

A. Einstein, W. Pauli, Ann. Math. 44, 131-137 (1943).

[34] S. W. Hawking, R. Penrose, Proc. R. Soc. Lond. Ser. A 314, 529-548 (1970).

[35] S. W. Hawking, G. F. R. Ellis, The Large Scale Structure of Space-Time. Cambridge University Press (1973).

[36] R. Penrose, Techniques of Differential Topology in Relativity. Society for Industrial and Applied Mathematics (1972).

[37] J. M. M. Senovilla, Gen. Rel. Grav. 30, 701-848 (1998) and references therein;

J. M. M. Senovilla, D. Garfinkle, Class. Quant. Grav. 32, 124008 (2015) and references therein.

[38] N. Tsitverblit, arXiv:1306.5997 (2013).

[39] E. B. Gliner, Sov. Phys. JETP 22, 378-382 (1966);

E. B. Gliner, Sov. Phys. Dokl. 15, 559-561 (1970).

[40] As seen for the initial Friedmann universe [38], transition from matter to its vacuum phase implies the presence of equal amounts for matter and antimatter. As also (admittedly) in that universe, such a phase transition thus ought to be preceded by the transition from a nonrelativistic matter to its radiation phase, where matter and antimatter are identical. 
[41] I. Dymnikova, Gen. Rel. Grav. 24, 235-242 (1992);

P. O. Mazur, E. Mottola, Proc. Nat. Acad. Sci. USA 101, 9545-9550 (2004);

M. Visser, D. L. Wiltshire, Class. Quant. Grav. 21, 1135-1151 (2004).

[42] R. Penrose, Rev. Mod. Phys. 37, 215-220 (1965);

P. Szekeres, Nature 228, 1183-1184 (1970);

K. A. Khan, R. Penrose, Nature 229, 185-186 (1971);

P. Szekeres, J. Math. Phys. 13, 286-294 (1972);

F. J. Tipler, Phys. Rev. D. 22, 2929-2932 (1980);

H. Bondi, F. A. E. Pirani, Proc. R. Soc. Lond. Ser. A 421, 395-410 (1989);

W. B. Bonnor, J. B. Griffiths, M. A. H. MacCallum, Gen. Rel. Grav. 26, 687-729 (1994) and references therein;

J. B. Griffiths, Colliding Plane Waves in General Relativity. Oxford University Press (1991); Dover Publications, New York (2016) and references therein.

[43] A. H. Taub, Ann. Math. 53, 472-490 (1951).

[44] W. B. Bonnor, J. Math. Mech. 6, 203-214 (1957) and references therein.

[45] A. Lichnerowicz, Théories relativistes de la gravitation et de l'électromagnétisme. Paris, Masson (1955).

[46] R. M. Wald, P. Yip, J. Math. Phys. 22, 2659-2665 (1981); J. Math. Phys. 23, 693 (1982).

[47] In empty space-time, the synchronous frame is comoving with a (pressureless) test matter.

[48] L. P. Eisenhart, Trans. Am. Math. Soc. 26, 205-220 (1924).

[49] R. Arnowitt, S. Deser, Ann. Phys. 23, 318-329 (1963) and references therein.

[50] With the geodesic deviation equation $[12,15,17,18,19]$, an infinitesimal deflection from a geodesic (parameterizable to be everywhere orthogonal to such a deflection [15]) could eventually vanish along the geodesic only when the Riemann tensor does not vanish there.

[51] A. Staruszkiewicz, Acta Phys. Pol. 24, 735-740 (1963);

J. R. Gott III, M. Alpert, Gen. Rel. Grav. 16, 243-247 (1984);

S. Giddings, J. Abbott, K. Kuchař, Gen. Rel. Grav. 16, 751-775 (1984);

S. Deser, R. Jackiw, G. 't Hooft, Ann. Phys. 152, 220-235 (1984);

see also the corresponding references in the works provided in Ref. [52].

[52] G. 't Hooft, Found. Phys 38, 733-757 (2008) and references therein;

M. van de Meent, Ph. D. thesis, Utrecht Univ., arXiv:1111.6468 (2011) and references therein.

[53] F. I. Cooperstock, Found. Phys. 22, 1011-1024 (1992);

F. I. Cooperstock, Ann. Phys. 282, 115-137 (2000);

F. I. Cooperstock, S. Tieu, Found. Phys. 33, 1033-1059 (2003).

[54] J. H. Taylor, L. A. Fowler, P. M. McCulloch, Nature 277, 437-440 (1979);

J. H. Taylor, J. M. Weisberg, Astrophys. J. 253, 908-920 (1982);

T.-P. Cheng, Relativity, Gravitation, and Cosmology. A basic introduction. Chapter 13. Oxford University Press (2005);

M. P. Hobson, G. P. Efstathiou, A. N. Lasenby, General Relativity. An Introduction for Physicists. Chapters 17 and 18. Cambridge University Press (2006);

J. M. Weisberg, D. J. Nice, J. H. Taylor, Astrophys. J. 722, 1030-1034 (2010) and references therein;

D. Kennefick, arXiv:1407.2164 (2014) and references therein;

the quadrupole approximation itself [9] is also discussed in [12, 13, 15, 17, 18, 19]. 
[55] D. Kennefick, arXiv:gr-qc/9704002 (1997) and references therein;

D. Kennefick, Traveling at the Speed of Thought. Einstein and the Quest for Gravitational Waves. Princeton University Press (2007) and references therein.

[56] A. Einstein, N. Rosen, J. Franklin Inst. 223, 43-54 (1937);

N. Rosen, Bull. Res. Counc. Israel 3, 328-332 (1954);

N. Rosen, Helv. Phys. Acta 29, Suppl. 4, 171-175 (1956);

J. Weber, J. A. Wheeler, Rev. Mod. Phys. 29, 509-515 (1957);

H. Bondi, F. A. E. Pirani, I. Robinson, Proc. R. Soc. Lond. Ser A 251, 519-533 (1959).

[57] L. Infeld, J. Plebanski, Motion and Relativity. Pergamon Press (1960) and references therein.

[58] F. A. E. Pirani, In: Gravitation: An Introduction to Current Research. Edited by L. Witten, pp. 199-226 (John Wiley \& Sons 1962) and references therein;

W. B. Bonnor, Brit. J. Appl. Phys. 14, 555-562 (1963) and references therein.

[59] K. S. Thorne, In: 300 Years of Gravitation. Edited by S. W. Hawking, W. Israel, pp. 330-458 (Cambridge University Press 1987);

S. Bonazzola, J.-A. Marck, Annu. Rev. Nucl. Part. Sci. 45, 655-717 (1994);

K. S. Thorne, arXiv:gr-qc/9704042 (1997);

B. S. Sathyaprakash, B. F. Schutz, Liv. Rev. Rel. 12, 2 (2009);

B. P. Abbott et al. (LIGO Scientific Collaboration and Virgo Collaboration), Liv. Rev. Rel. 19, 1 (2016);

B. P. Abbott et al. (LIGO Scientific Collaboration and Virgo Collaboration), Phys. Rev. Lett. 116, 061102 (2016);

B. P. Abbott et al. (LIGO Scientific Collaboration and Virgo Collaboration), Phys. Rev. D 93, 122003 (2016);

The Nobel Committee for Physics, Scientific Background on the Nobel Prize in Physics 2017 https://www.nobelprize.org/nobel_prizes/physics/laureates/2017/advanced-physicsprize2017.pdf; see also references in all these works.

[60] R. A. Isaacson, Phys. Rev. 166, 1263-1271 (1968);

R. A. Isaacson, Phys. Rev. 166, 1272-1280 (1968);

K. S. Thorne, In: Gravitational Radiation. Edited by N. Deruelle, T. Piran, pp. 1-57 (North Holland, Amsterdam 1983) and references therein;

E. E. Flanagan, S. A. Hughes, New J. Phys. 7, 204 (2005) and references therein;

M. Maggiore, Gravitational Waves. Vol. 1. Theory and Experiments. Chapter 1. Oxford University Press (2008) and references therein.

[61] S. Deser, J. Franklin, Am. J. Phys. 73, 261-264 (2005) and references therein.

[62] For such a noninertial reference frame as say that of a rotating disk [17], space-time curvature is due to the Lorentz contraction (in the inertial frame at rest) being relevant only to the disk circumference. The ratio of the latter to the (invariant) disk radius would then have to exceed $2 \pi$. Such a curvature could however be physically meaningful only when the disk is a real material object. In particular, the principle of equivalence seems to implicitly amount the space-time curvature arising when matter is relatively accelerated in a conventional (materially defined) interaction to that of empty space-time as such. Essentially suggesting the availability of what ought to be viewed as a mechanism of "distant action", the equivalence thus assumed gives rise to the above apparent paradox in interpretation of the Einstein field equations. That space-time curvature underlying the theory of general relativity may actually have to imply the existence of a material background for the physical manifestation of such a curvature was also discussed by A. Einstein himself in [63]. 
[63] A. Einstein, In: Sidelights on Relativity. Translated by G. B. Jeffery, W. Perrett, pp. 3-24 (Methuen \& Co. Ltd., London 1922; Springer, Berlin 1920 in German) http://www.archive.org/details/sidelightsonrela00einsuoft; also in The Genesis of General Relativity. Edited by M. Janssen, J. D. Norton, J. Renn, T. Sauer, J. Stachel, pp. 1537-1542 (Boston Studies in the Philosophy of Science 250, Springer 2007).

[64] J. A. S. Lima, Phys. Rev. D 54, 2571-2577 (1996).

[65] J. A. Frieman, M. S. Turner, D. Huterer, Annu. Rev. Astron. Astrophys. 46, 385-432 (2008).

[66] S. Carlip, Class. Quant. Grav. 25, 154010 (2008);

S. Boughn, Found. Phys. 39, 331-351 (2009);

Y. K. Ha, Int. J. Mod. Phys.: Conf. Ser. 7, 219-226 (2012);

Y. K. Ha, J. Phys.: Conf. Ser. 484, 012061 (2014);

see also references in all these works.

[67] Gravitation arising in [52] from space-time defects created by strings has a singular nature.

[68] Assigning a material significance to the infinite boundaries of space-time, the existence of matter at such a boundary ought to amount to a singular behavior. One could still nonsingularly assume such an existence outside any finite space-time region, i.e. for the respective open infinite space-time. Since discontinuity in space-time curvature at the matter boundary underlies the present view on general relativity, this has to be legitimate.

[69] E. Noether, Nachr. König. Gesellsch. Wiss. Gött., Math.-phys. Klasse, 235-257 (1918); English translation by M. A. Tavel, arXiv:physics/0503066v2 (2015);

Y. Kosmann-Schwarzbach, The Noether Theorems. Invariance and Conservations Laws in the Twentieth Century. Translated by B. E. Schwarzbach. Springer (2011), for English translation of the Noether paper, see pp. 3-22.

[70] D. E. Neuenschwander, Emmy Noether's Wonderful Theorem. Johns Hopkins University Press (2011).

[71] The pressure, if any, is implied to be related to the energy density by a finite number.

[72] The understanding of general relativity suggested herein arises from the generalized principle of relativity alone, when this postulate is applied to a material system whose elements are connected at least by the background medium. As opposed to the view that space-time as such could possess curvature, it could thus be potentially consistent with certain interpretations of what is referred to as Mach's principle [12, 13, 15, 18, 19]. Any matter source itself thus defines a reference frame associated with it and would then have to contribute to the relative space-time curvature affecting all other such sources. A detailed discussion of Mach's principle and its relevant interpretations is however outside the present scope.

[73] Standing for the so-called passive gravitational density as well, the inertial density of a perfect fluid complying with Eqs. (1) arises from the contracted differential Bianchi identities as $\rho+p$ [48], where $\rho$ is the fluid energy density and $p$ is the pressure. The corresponding active gravitational density is however $\rho+3 p$, as suggested say by the Friedmann acceleration equation. Since these densities do not coincide, the conventional third law of Newton does not generally apply to gravitation. This could be attributed to the gravitation-related space-time curvature arising within the background medium independently of a conventional interaction between different matter sources, although such a curvature is still a part of the overall energy-momentum balance with its sources.

[74] The comma and semicolon denote the ordinary and covariant differentiations, respectively. 
[75] Apart from spatially compact $\Omega$, as assumed in Sec. 4.1, this also applies when $\Omega$ (filled with matter) is generally an open infinite space-time [68]. The integrands of Eq. (8), $\delta g^{i j}$ in Eqs. (10), as well as $G_{i}^{j}$ and $T_{i}^{j}$ then still vanish in the limit at $\partial \Omega$.

[76] This applies to the derivation of the Einstein field equations themselves as well. In many courses on general relativity, such a derivation from the action principle seems to suggest that the first derivatives of the metric tensor in the directions normal to the hypersurface serving as the boundary of the integration area also have to be explicitly prescribed at this boundary, apart from the metric tensor itself. This issue is recognized in (Appendix E of Ref.) [15] as well, where an extra term is thus added to the gravitational action to cancel the nonzero contribution from the boundary when the metric tensor alone is prescribed there. From the present perspective on general relativity, the boundary features due to which the second derivatives of the metric tensor in the Lagrangian density for gravitation are rendered irrelevant arise naturally from the space-time change to its flatness at $\partial \Omega$. This takes place when $\Omega$ (filled with matter) is either just spatially compact or such as generally stands for an open infinite space-time [68] that becomes flat in the limit at $\partial \Omega$.

[77] Eqs. (17) could also result either from Eqs. (1) alone or from Eqs. (1) and (15) when Eqs. (15) arise from the scalar invariance of $S_{T}$. Unless treated so already in Eqs. (1), however, $G_{i}^{j} / \kappa$ is not then viewable as the gravitation energy-momentum tensor yet. Unlinked to the background flatness, in particular, such procedures are not unequivocally defined.

[78] Eqs. (17) result from Eqs. (1) and (15) when Eqs. (15) arise from the scalar invariance of $S_{T}$ as well [77]. Although Eqs. (17) coincide with the ordinary divergence of Eqs. (1), the second Noether theorem [16, 69] then still fails to turn Eqs. (17) into an improper conservation law. As the Euler-Lagrange equations [15, 18] for a materially defined field of space-time curvature, in particular, Eqs. (1) do not yet qualify as also such equations for the field of matter itself. With the material nature of space-time curvature, Eqs. (15) actually arise in Sec. 4.2 from flatness of the space-time background. For such a separate invariance, Eqs. (17) come from Eqs. (1) and (15) as the proper conservation law implied by the first Noether theorem [16, 69, 70]. This law is thus what underlies Eqs. (1) as well.

[79] T. Jacobson, Phys. Rev. Lett. 75, 1260-1263 (1995);

T. Padmanabhan, Phys. Rep. 406, 49-125 (2005);

T. Padmanabhan, Rep. Prog. Phys. 73, 046901 (2010);

E. Verlinde, J. High Energy Phys. 04 (2011) 029;

T. Padmanabhan, Res. Astron. Astrophys. 12, 891-916 (2012);

T. Padmanabhan, Gen. Rel. Grav. 46, 1673 (2014);

T. Padmanabhan, Curr. Sci. 109, 2236-2242 (2015).

[80] J. D. Bekenstein, Phys. Rev. D 7, 2333-2346 (1973);

J. M. Bardeen, B. Carter, S. W. Hawking, Comm. Math. Phys. 31, 161-170 (1973);

S. W. Hawking, Comm. Math. Phys. 43, 199-220 (1975);

G. W. Gibbons, S. W. Hawking, Phys. Rev. D 15, 2738-2751 (1977);

G. 't Hooft, arXiv:gr-qc/9310026 (1993);

L. Susskind, J. Math. Phys. 36, 6377-6396 (1995);

R. M. Wald, Liv. Rev. Rel. 4, 6 (2001);

R. Bousso, Rev. Mod. Phys. 74, 825-874 (2002).

[81] R. Penrose, Ann. N. Y. Acad. Sci. 571, 249-264 (1989) and references therein.

[82] Y. B. Zeldovich, Sov. Phys. Usp. 11, 381-393 (1968).

[83] A. D. Sakharov, Sov. Phys. JETP 52, 349-351 (1980);

R. Peierls, Phys. Today 47(11), 115-115 (1994). 